\title{
First-Line Biological Agents Plus Chemotherapy in Older Patients with Metastatic Colorectal Cancer: A Retrospective Pooled Analysis
}

\author{
Pilar García-Alfonso' ${ }^{1}$ (1) Eduardo Díaz-Rubio ${ }^{2}$ - Albert Abad ${ }^{3,4}$ - Alfredo Carrato ${ }^{5}$ - Bartomeu Massutí ${ }^{6}$. \\ María José Ortiz-Morales ${ }^{7}$. José Luis Manzano Mozo ${ }^{3}$. Andrés Muñoz ${ }^{1}$. Gema Durán ${ }^{8}$. Javier Sastre ${ }^{2}$. \\ María José Safont ${ }^{9} \cdot$ Reyes Ferreiro ${ }^{5}$. Fernando Rivera ${ }^{10}$. Encarnación González ${ }^{11}$. Manuel Valladares-Ayerbes ${ }^{12}$. \\ Cristina Grávalos ${ }^{13}$. Vicente Alonso-Orduña ${ }^{14}$. José María Viéitez ${ }^{15}$. Alfonso Yubero ${ }^{16}$. Enrique Aranda ${ }^{7}$ on behalf \\ of the Spanish Cooperative Group for the Treatment of Digestive Tumors (TTD)
}

Accepted: 7 January 2021 / Published online: 22 February 2021

(c) The Author(s) 2021

\begin{abstract}
Background Biologicals, in combination with chemotherapy, are recommended as first-line treatment of metastatic colorectal cancer (mCRC); however, evidence guiding the appropriate management of older patients with mCRC is limited.

Objective This study was undertaken to compare the efficacy and safety outcomes in older versus younger patients with mCRC who received first-line biological therapy.

Methods This retrospective analysis used pooled data from five trials undertaken by the Spanish Cooperative Group for the Treatment of Digestive Tumours. All were studies of adults with advanced CRC who received first-line treatment with chemotherapy plus bevacizumab, cetuximab or panitumumab, stratified by age ( $\geq 65$ vs. $<65$ years). Endpoints included progression-free survival (PFS), overall survival (OS), overall response rate (ORR) and safety.

Results In total, 999 patients from five studies were included in the analysis: $480(48 \%)$ were aged $\geq 65$ years, and 519 (52\%) were aged $<65$ years. Median PFS did not differ significantly between patients aged $\geq 65$ and $<65$ years $(9.9$ vs. 9.4 months; hazard ratio [HR] 1.01; 95\% confidence interval [CI] 0.88-1.17). Median OS was significantly shorter in older than in younger patients ( 21.3 vs. 25.0 months; HR 1.21; 95\% CI 1.04-1.41). There was no significant difference between older and younger patients in ORR ( 59 vs. $62 \%$ ). Patients aged $\geq 65$ years experienced significantly more treatment-related grade 3 or higher adverse events $(61.67 \%)$ than did patients aged $<65$ years $(45.86 \%)$.

Conclusions Biologicals plus chemotherapy is an effective first-line treatment option for selected patients aged $\geq 65$ years with $\mathrm{mCRC}$ and has a manageable safety profile and efficacy comparable to that observed in younger patients.
\end{abstract}

\section{Introduction}

Colorectal cancer (CRC) is a common cause of morbidity and mortality, particularly in older people [1]. In the USA, over $50 \%$ of new CRC diagnoses are in patients aged $\geq 65$ years [2]. Older patients with CRC have a higher percentage of comorbidities and organ function impairment and are at higher risk of developing treatment-related toxicities.

The collaborator names of the Spanish Cooperative Group for the Treatment of Digestive Tumors (TTD) are present in the Acknowledgements section.

Pilar García-Alfonso

pgarcaalfonso@gmail.com

Extended author information available on the last page of the article
For most patients with unresectable metastatic CRC (mCRC), clinical guidelines recommend first-line treatment with biological agents (i.e. targeted monoclonal antibody therapy) in combination with cytotoxic chemotherapy [3]. However, treatment guidelines are generally based on the results of randomized clinical trials rather than real-world data, and the inclusion and exclusion criteria used in such studies mean that older patients are often under-represented in clinical trials [4]. An analysis of National Cancer Institute-sponsored clinical trials (active from 1997 to 2000) showed that, although $61 \%$ of newly diagnosed CRC in the USA was in patients aged $\geq 65$ years, only $32 \%$ of them participated in clinical trials [5]. Thus, evidence guiding the appropriate management of older patients with mCRC is incomplete, making it unclear to physicians as to whether 


\section{Key Points}

It is unclear whether treatment with a biological agent plus chemotherapy $(\mathrm{B}+\mathrm{C})$, which is generally recommended as first-line treatment for metastatic colorectal cancer (mCRC), is optimal in older patients.

This pooled analysis of data from five clinical trials examined the efficacy and safety of first-line B $+\mathrm{C}$ treatment for mCRC according to age group ( $<65$ years vs. $\geq 65$ years).

We found that selected older patients, mainly aged $<75$ years, may be confidently treated with a first-line B $+C$ regimen and that efficacy in this population is comparable to that seen in younger adults.

Toxicity is increased in older patients, but adverse events can usually be anticipated and managed without treatment discontinuation.

their older patients can be managed in the same way as younger patients.

In 2020, the French Society of Geriatric Oncology published the first guidelines on the treatment of mCRC in older patients since 2013 [6]. Despite the publication of several clinical trials in the intervening years, only one grade A recommendation was made; this was in support of first-line bevacizumab in combination with mono-chemotherapy. All other recommendations relating to the use of biological agents were of a lower grade or were based on expert opinion only. No recommendations were made regarding the use of specific agents other than bevacizumab.

The Spanish Cooperative Group for the Treatment of Digestive Tumours (TTD) has sponsored several trials of biological therapy in patients with mCRC. Here, we report a retrospective analysis of data from these published clinical trials, where the percentage of the population aged $>$ 65 years was $48 \%$, which compared efficacy and safety outcomes in older versus younger patients with mCRC.

\section{Patients and Methods}

\subsection{Study Design}

A retrospective subgroup analysis according to age $(<$ 65 vs. $\geq 65$ years) was conducted using a database of adults with advanced mCRC who were enrolled in five trials sponsored by the TTD. In these trials, patients were receiving first-line treatment with standard chemotherapy plus targeted monoclonal antibody therapy (bevacizumab, cetuximab or panitumumab). The design and results of the trials have been previously published (Table $\mathrm{S} 1$ in the electronic supplementary material [ESM]) [7-11]. Briefly, all trials were multicentre and open-label; four were phase II [7-9, 11] and one was a phase III trial [10]. One phase II trial was a single-arm study [7], whereas patients in the other four trials were randomized to two treatment arms (Table S1 in the ESM) [8-11]. One phase II trial included only patients with wild-type Kirsten rat sarcoma virus proto-oncogene (KRAS) mCRC and multiple or unresectable liver-limited disease [9], and another included only patients with wild-type KRAS mCRC (Table $\mathrm{S} 1$ in the ESM) [8]. All studies were conducted in accordance with the Declaration of Helsinki and were approved by institutional review boards or independent ethics committees at each centre. All patients gave written informed consent.

Analysed efficacy endpoints included progression-free survival (PFS), overall survival (OS), overall response rate (ORR; defined as the proportion of patients who achieved either complete response [CR] or a partial response [PR] to treatment) and disease control rate (DCR; defined as the proportion of patients with CR, PR or stable disease). Safety was evaluated according to National Cancer Institute Common Toxicity Criteria, versions 4 and 5.

\subsection{Statistical Analyses}

The Chi-squared test was used to compare the baseline clinical characteristics and the percentage of patients responding to treatment in the two age groups. PFS and OS were determined using the Kaplan-Meier method, and the logrank test and Cox regression model were employed for the comparison of the curves. The Chi-squared test was also used to compare the two age groups for safety, treatment delays, dose reductions and dose intensity. A more detailed secondary analysis of the impact of age on OS and PFS was undertaken by analysing these parameters in patients aged $<65$ years, $\geq 65$ to $<70$ years, $\geq 70$ to $<75$ years, and $\geq$ 75 years.

\section{Results}

\subsection{Patient Characteristics}

Data were analysed for a total of 999 patients from the five studies (Table 1). The majority of patients were male $(64.06 \%)$, and the mean age was 63.27 years. 
In the pooled population of patients from the five studies, $48.05 \%$ of patients were aged $\geq 65$ years. In MACRO2, which excluded patients aged $>70$ years, the proportion of patients aged $\geq 65$ years was lower than in the other four studies (26.46 vs. 48.33-55.46\%; Table 1). In the total population, $19.42 \%$ of patients were aged $\geq 65$ to $<70$ years, $16.92 \%$ were aged $\geq 70$ to $<75$ years and $11.71 \%$ were $\geq$ 75 years (Table 1 ).

When comparing the clinical characteristics of patients aged $<65$ versus $\geq 65$ years (Table 2 ), significantly more patients aged $\geq 65$ years ( 42.08 vs. $31.41 \%$; $p=0.0005$ ) and significantly more patients aged $<65$ years $(74.15$ vs. $63.22 \% ; p=0.0005$ ) had lung metastases, whereas there was no significant difference in $R A S$ mutation status. As expected, there was also a significant difference between groups ( $<65$ vs. $\geq 65$ years) in the proportion of patients with a baseline Eastern Cooperative Oncology Group performance status (ECOG PS) score of 0 (29.92 vs. 19.00\%; $p=0.0005$; Table 2). The primary tumour location was the colon for the majority of patients in both age groups, with no significant between-group difference; however, the primary tumour site was the left colon for significantly more patients aged $\geq 65$ versus $<65$ years ( 84.09 vs. $74.16 \%$, $p=0.0390$; Table 2). Significantly more patients aged $\geq 65$ years had previously undergone surgery (82.92 vs. $74.37 \% ; p=0.0010$; Table 2). In terms of metastases, most patients in both age groups had up to two organs involved, with lung metastases present in significantly more patients aged $\geq 65$ than $<65$ years ( 42.08 vs. $31.41 \%, p=0.0005$; Table 2).
All patients had at least one comorbidity (range 1-15); the median number of comorbidities per patient was 2.0 both in patients aged $<65$ years and in those aged $\geq$ 65 years. The most common comorbidities in both age groups were hypertension (present in 27.36 and $48.13 \%$ of patients aged $<65$ vs. $\geq 65$ years, respectively), dyslipidaemia (present in 13.68 and $22.29 \%$, respectively) and diabetes (present in 13.87 and $21.88 \%$, respectively).

\subsection{Treatment}

Of the 999 patients included in this analysis, 733 $(73.37 \%)$ were treated with bevacizumab, $189(18.92 \%)$ received cetuximab and $77(7.71 \%)$ received panitumumab (Table 2).

The use of biological agents differed significantly between the two age groups $(p<0.0001)$. In patients aged $\geq 65$ years, $392(81.67 \%)$ received bevacizumab, $50(10.42 \%)$ received cetuximab and $38(7.92 \%)$ panitumumab; in those aged < 65 years, 341 (65.70\%), 139 $(26.78 \%)$ and $39(7.51 \%)$ patients received the respective agents (Table 2).

The median treatment duration in patients aged $<$ 65 years and $\geq 65$ years was 18.57 (interquartile range [IQR] 17.86-23.00) and 18.14 (IQR 13.14-20.79) weeks, respectively, for bevacizumab, 18.29 (IQR 11.00-26.71) and 18.57 (IQR 10.71-25.00) weeks, respectively, for panitumumab and 15.29 (IQR 10.14-17.14) and 15.21 (IQR 7.29-17.14) weeks, respectively, for cetuximab.

The mean \pm standard deviation $(\mathrm{SD})$ relative dose intensity of panitumumab was significantly higher in

Table 1 Age distribution at baseline by study

\begin{tabular}{|c|c|c|c|c|c|c|}
\hline & $\begin{array}{l}\text { AVAXIRI [7] } \\
(N=77)\end{array}$ & $\begin{array}{l}\text { MACRO2 } \\
(N=189)^{\mathrm{a}}\end{array}$ & $\begin{array}{l}\text { PLANET[9] } \\
(N=77)\end{array}$ & $\begin{array}{l}\text { MACRO [10] } \\
(N=476)\end{array}$ & $\begin{array}{l}\text { SETICC [11] } \\
(N=180)\end{array}$ & $\begin{array}{l}\text { Total } \\
(N=999)\end{array}$ \\
\hline \multicolumn{7}{|l|}{ Age, years } \\
\hline Mean \pm SD & $64.82 \pm 8.55$ & $59.06 \pm 8.33$ & $61.78 \pm 11.17$ & $64.74 \pm 9.56$ & $63.78 \pm 9.82$ & $63.27 \pm 9.68$ \\
\hline Median (range) & $\begin{array}{l}65.00(41.00- \\
81.00)\end{array}$ & $\begin{array}{l}60.00(33.00- \\
73.00)\end{array}$ & $\begin{array}{l}64.00(32.00- \\
83.00)\end{array}$ & $\begin{array}{l}66.00(32.00- \\
83.00)\end{array}$ & $\begin{array}{l}64.00(29.00- \\
86.00)\end{array}$ & $\begin{array}{l}64.00(29.00- \\
86.00)\end{array}$ \\
\hline \multicolumn{7}{|l|}{ Age groups, years } \\
\hline$<65$ & $36(46.75)$ & $139(73.54)$ & $39(50.65)$ & $212(44.54)$ & $93(51.67)$ & $519(51.95)$ \\
\hline$\geq 65$ & $41(53.25)$ & $50(26.46)$ & $38(49.35)$ & $264(55.46)$ & $87(48.33)$ & $480(48.05)$ \\
\hline \multicolumn{7}{|l|}{$\begin{array}{l}\text { Detailed age } \\
\text { groups, years }\end{array}$} \\
\hline$<65$ & $36(46.75)$ & $139(73.54)$ & $39(50.65)$ & $212(44.54)$ & $93(51.67)$ & $519(51.95)$ \\
\hline$\geq 65$ to $<70$ & $19(24.68)$ & $40(21.16)$ & $19(24.68)$ & $87(18.28)$ & $29(16.11)$ & $194(19.42)$ \\
\hline$\geq 70$ to $<75$ & $13(16.88)$ & $10(5.29)$ & $9(11.69)$ & $104(21.85)$ & $33(18.33)$ & 169 (16.92) \\
\hline$\geq 75$ & $9(11.69)$ & 0 & $10(12.99)$ & $73(15.34)$ & $25(13.89)$ & $117(11.71)$ \\
\hline
\end{tabular}

Data are presented as $N(\%)$ unless otherwise specified

$S D$ standard deviation

${ }^{\mathrm{a}}$ MACRO2 excluded patients aged $>70$ years 
Table 2 Clinical characteristics and treatments at baseline, by age group

\begin{tabular}{|c|c|c|c|c|}
\hline Variable & $<65$ years $(N=519)$ & $\geq 65$ years $(N=480)$ & Total $(N=999)$ & $p$ value \\
\hline ECOG PS ${ }^{\mathrm{a}}$ & $N=518$ & $N=479$ & $N=997$ & \\
\hline 0 & $155(29.92)$ & $91(19.00)$ & $246(24.67)$ & $0.0005^{\mathrm{b}}$ \\
\hline 1 & $249(48.07)$ & $268(55.95)$ & $517(51.86)$ & \\
\hline 2 & $107(20.66)$ & $117(24.43)$ & $224(22.47)$ & \\
\hline 3 & $7(1.35)$ & $3(0.63)$ & $10(1.00)$ & \\
\hline \multicolumn{5}{|l|}{ Primary tumour location } \\
\hline Colon & $336(67.74)$ & $284(59.17)$ & $620(62.06)$ & $0.0916^{\mathrm{c}}$ \\
\hline Rectum & $124(23.89)$ & $144(30.00)$ & $268(26.83)$ & \\
\hline Colon and rectum & $59(11.37)$ & $52(10.83)$ & $111(11.11)$ & \\
\hline Primary tumour location ${ }^{\mathrm{a}}$ & $N=178$ & $N=88$ & $N=266$ & \\
\hline Left & $132(74.16)$ & $74(84.09)$ & $206(77.44)$ & $0.0390^{\mathrm{b}}$ \\
\hline Right & 37 (20.79) & $14(15.91)$ & $51(19.17)$ & \\
\hline Unknown & $9(5.06)$ & 0 & $9(3.38)$ & \\
\hline \multicolumn{5}{|l|}{ Metastatic site } \\
\hline Liver & $421(81.12)$ & $391(81.46)$ & $812(81.28)$ & $0.8903^{\mathrm{c}}$ \\
\hline Lungs & $163(31.41)$ & $202(42.08)$ & $365(36.54)$ & $0.0005^{\mathrm{c}}$ \\
\hline Bone & $7(1.35)$ & $8(1.67)$ & $15(1.50)$ & $0.6797^{\mathrm{c}}$ \\
\hline Peritoneum & $57(10.98)$ & $46(9.58)$ & $103(10.31)$ & $0.4674^{\mathrm{c}}$ \\
\hline Distant lymph nodes & $70(13.49)$ & $63(13.13)$ & $133(13.31)$ & $0.8662^{c}$ \\
\hline \multicolumn{5}{|l|}{ Number of organs involved } \\
\hline$\leq 2$ & $492(94.80)$ & $446(92.92)$ & $938(93.89)$ & $0.2148^{\mathrm{c}}$ \\
\hline$>2$ & $27(5.20)$ & $34(7.08)$ & $61(6.11)$ & \\
\hline$R A S$ status $^{\mathrm{a}}$ & $N=156$ & $N=75$ & $N=231$ & \\
\hline Mutated & $25(16.03)$ & $18(24.00)$ & $43(18.61)$ & $0.1526^{\mathrm{b}}$ \\
\hline$K R A S$ status $^{\mathrm{a}}$ & $N=472$ & $N=416$ & $N=888$ & \\
\hline Mutated & $122(25.85)$ & $153(36.78)$ & $275(30.97)$ & $0.0005^{\mathrm{b}}$ \\
\hline Wild type & $350(74.15)$ & $263(63.22)$ & $613(69.03)$ & $0.0005^{\mathrm{b}}$ \\
\hline \multicolumn{5}{|l|}{ Biological agent } \\
\hline Bevacizumab & $341(65.70)$ & $392(81.67)$ & $733(73.37)$ & $<0.0001^{\mathrm{c}}$ \\
\hline Cetuximab & $139(26.78)$ & $50(10.42)$ & $189(18.92)$ & \\
\hline Panitumumab & $39(7.51)$ & $38(7.92)$ & $77(7.71)$ & \\
\hline \multicolumn{5}{|l|}{ Chemotherapy agent } \\
\hline 5-Fluorouracil & 207 (39.88) & $111(23.13)$ & $318(31.83)$ & \\
\hline Capecitabine & $312(60.12)$ & $369(76.88)$ & $681(68.17)$ & $<0.0001^{\mathrm{c}, \mathrm{d}}$ \\
\hline Irinotecan & $100(19.27)$ & $89(18.54)$ & $189(18.92)$ & \\
\hline Oxaliplatin & $419(80.73)$ & $391(81.46)$ & $810(81.08)$ & $0.7697^{\mathrm{c}, \mathrm{e}}$ \\
\hline \multicolumn{5}{|l|}{ Previous treatment } \\
\hline Surgery & $386(74.37)$ & $398(82.92)$ & $784(78.48)$ & $0.0010^{\mathrm{c}}$ \\
\hline Chemotherapy & $69(13.29)$ & $80(16.67)$ & $149(14.91)$ & $0.1350^{c}$ \\
\hline Radiotherapy & $34(6.55)$ & $40(8.33)$ & $74(7.41)$ & $0.2825^{\mathrm{c}}$ \\
\hline \multicolumn{5}{|l|}{ Comorbidities $^{\mathrm{f}}$} \\
\hline Arthropathy & $31(5.97)$ & $42(8.75)$ & $73(7.31)$ & 0.1133 \\
\hline Benign prostatic hyperplasia & $15(2.89)$ & $42(8.75)$ & $57(5.71)$ & $<0.0001$ \\
\hline Diabetes & $72(13.87)$ & $105(21.88)$ & $177(17.72)$ & 0.0012 \\
\hline Dyslipidaemia & $71(13.68)$ & $107(22.29)$ & $178(17.82)$ & 0.0005 \\
\hline Hypertension & $142(27.36)$ & $231(48.13)$ & $373(37.34)$ & $<0.0001$ \\
\hline
\end{tabular}

Data are presented as $N(\%)$ unless otherwise indicated. $p<0.05$ is considered statistically significant

ECOG PS Eastern Cooperative Oncology Group performance status

${ }^{\text {a}}$ Percentages were calculated based on the number of patients with data available for these variables

${ }^{\text {b}}$ Fisher's exact test

${ }^{\mathrm{c}}$ Chi-squared test

${ }^{\mathrm{d}}$ Versus 5-fluorouracil

${ }^{\mathrm{e}}$ Versus irinotecan

${ }^{\mathrm{f}}$ Only comorbidities occurring in $>5 \%$ of patients overall are shown 
patients aged $<65$ than in those $\geq 65$ years $(87.20 \pm$ $13.18 \%$ vs. $82.80 \pm 11.21 \% ; p=0.0372$ ), most likely because the relative dose intensity of panitumumab was reduced in patients aged $\geq 75$ versus $<75$ years (Table 3 ).

There were no significant differences between age groups in the mean \pm SD relative dose intensity of bevacizumab $(90.34 \pm 14.33 \%$ vs. $90.75 \pm 13.71 \% ; p=0.8228)$ or cetuximab $(86.92 \pm 11.10 \%$ vs. $84.85 \pm 15.21 \%$; $p=$ $0.6612)$.

There were no significant differences between those aged $<65$ and $\geq 65$ years in the incidence of biological drug dose reductions $(55.30$ vs. $61.25 \% ; p=0.0568)$ or delays (60.12 vs. $57.50 \%$; $p=0.4013)$.

\subsection{Efficacy}

PFS did not differ significantly between the two age groups; median PFS was 9.9 months (95\% confidence interval [CI] 9.1-10.4) for patients aged $\geq 65$ years and 9.4 months $(95 \%$ CI 8.9-10.5) for those aged $<65$ years ( $p=0.8585$; hazard ratio [HR] 1.01; 95\% CI 0.88-1.17; Fig. 1a). In the detailed age groups, median PFS was 8.6 months (95\% CI 7.0-10.6) in patients aged $\geq 75$ years, 9.9 months $(95 \%$ CI $8.5-11.0)$ in those aged $\geq 70$ to $<75$ years, 9.9 months $(95 \%$ CI $8.9-11.3$ ) in those aged $\geq 65$ to $<70$ years and 9.4 months (95\% CI 8.9-10.5) in those aged $<65$ years (Fig. 2a).

Median OS was significantly shorter in patients aged $\geq 65$ years (21.3 months; 95\% CI 18.9-23.2) than in those aged < 65 years (25.0 months; 95\% CI 22.9-27.3; $p=0.0132$; HR $1.21 ; 95 \%$ CI 1.04-1.41; Fig. 1b). In the detailed age groups, median OS showed numerical decreases from 25.0 months in patients aged $<65$ years to 17.6 months in those aged $\geq$ 75 years (Fig. 2b). In univariate Cox regression analysis, age was a significant prognostic factor for OS but not for PFS.

Other efficacy endpoints, including best overall response, ORR and DCR, showed no significant differences between the two age groups (Table 4). The ORR was $58.96 \%$ in patients aged $\geq 65$ years and $62.04 \%$ in those aged $<65$ years. When non-evaluable patients were excluded from the analysis, the ORR was 64.32 and $65.58 \%$ for the respective age groups. The DCR was 84.38 and $87.28 \%$ in patients aged $\geq 65$ years and $<65$ years, respectively; respective DCR values were 92.05 and $92.26 \%$ when non-evaluable patients were excluded from the analysis.

Among patients receiving bevacizumab $(N=733)$, median PFS did not significantly differ between patients aged $<65$ versus $\geq 65$ years (9.7 vs. 9.7 months; $p=0.4250$ ). Consistent with the total population, median OS in patients receiving bevacizumab was significantly shorter in patients aged $\geq 65$ versus $<65$ years ( 20.0 vs. 23.8 months), with analysis of the detailed age groups showing that the shortest median OS was in those aged $\geq 75$ years ( 17.1 months). OS among patients receiving anti-epidermal growth factor receptors was similar for patients aged $<65$ and $\geq 65$ years (27.7 vs. 27.1 months; $p=0.9090$ ). The ORR in patients receiving bevacizumab was similar in the groups aged $<65$ and $\geq 65$ years ( 59.24 vs. $57.14 \% ; p=1.000)$.

\subsection{Safety}

The most common adverse events (AEs; i.e. those experienced by $>5 \%$ of patients in either treatment group) are shown in Table 5, with further detail in Tables S2 and S3 in the ESM. The incidence of fatigue, upper respiratory tract infection, decreased appetite and hypertension (any grade) were significantly higher in patients aged $\geq$ 65 years than in those aged $<65$ years (Table 5). Grade 3-5 fatigue and several gastrointestinal AEs (diarrhoea, nausea, rectal haemorrhage and vomiting) occurred in significantly more patients aged $\geq 65$ years than in those aged $<65$ years (Table 5).

AEs of any grade with significantly higher incidence in patients aged $<65$ versus $\geq 65$ years were anaemia, asthenia, skin toxicity dermatitis and skin fissures (Table 5). Grade 1-2 neutropenia was also significantly more common in the younger patient group. Skin toxicity dermatitis was the only grade $3-5$ AE that occurred with significantly higher incidence in patients aged $<65$ years.

The overall incidence of grade 3 or higher AEs was significantly higher in patients aged $\geq 65$ than $<65$ years

Table 3 Relative dose intensity of biological agents, by detailed age group

\begin{tabular}{llllll}
\hline & $\begin{array}{l}<65 \text { years } \\
(N=519)\end{array}$ & $\begin{array}{l}\geq 65 \text { to }<70 \text { years } \\
(N=194)\end{array}$ & $\begin{array}{l}\geq 70 \text { to }<75 \text { years } \\
(N=169)\end{array}$ & $\begin{array}{l}\geq 75 \text { years } \\
(N=117)\end{array}$ & $\begin{array}{l}\text { Total } \\
(N=999)\end{array}$ \\
\hline Bevacizumab & $N=341$ & $N=135$ & $N=150$ & $N=107$ & $N=733$ \\
Relative dose intensity, $\%$ & $90.34 \pm 14.33$ & $91.16 \pm 14.33$ & $90.24 \pm 14.27$ & $90.95 \pm 12.12$ & $90.56 \pm 13.99$ \\
Cetuximab & $N=132$ & $N=39$ & $N=10$ & $N=0$ & $N=181$ \\
Relative dose intensity, $\%$ & $86.92 \pm 11.10$ & $83.56 \pm 16.61$ & $90.35 \pm 5.30$ & - & $N=10$ \\
Panitumumab & $N=39$ & $N=19$ & $N=9$ & $76.39 \pm 12.33$ \\
Relative dose intensity, $\%$ & $87.20 \pm 13.18$ & $84.67 \pm 11.41$ & $84.01 \pm 9.22$ & $78.14 \pm 12.17$ & $85.03 \pm 12.37$ \\
\hline
\end{tabular}

Data are presented as mean \pm standard deviation unless otherwise indicated 

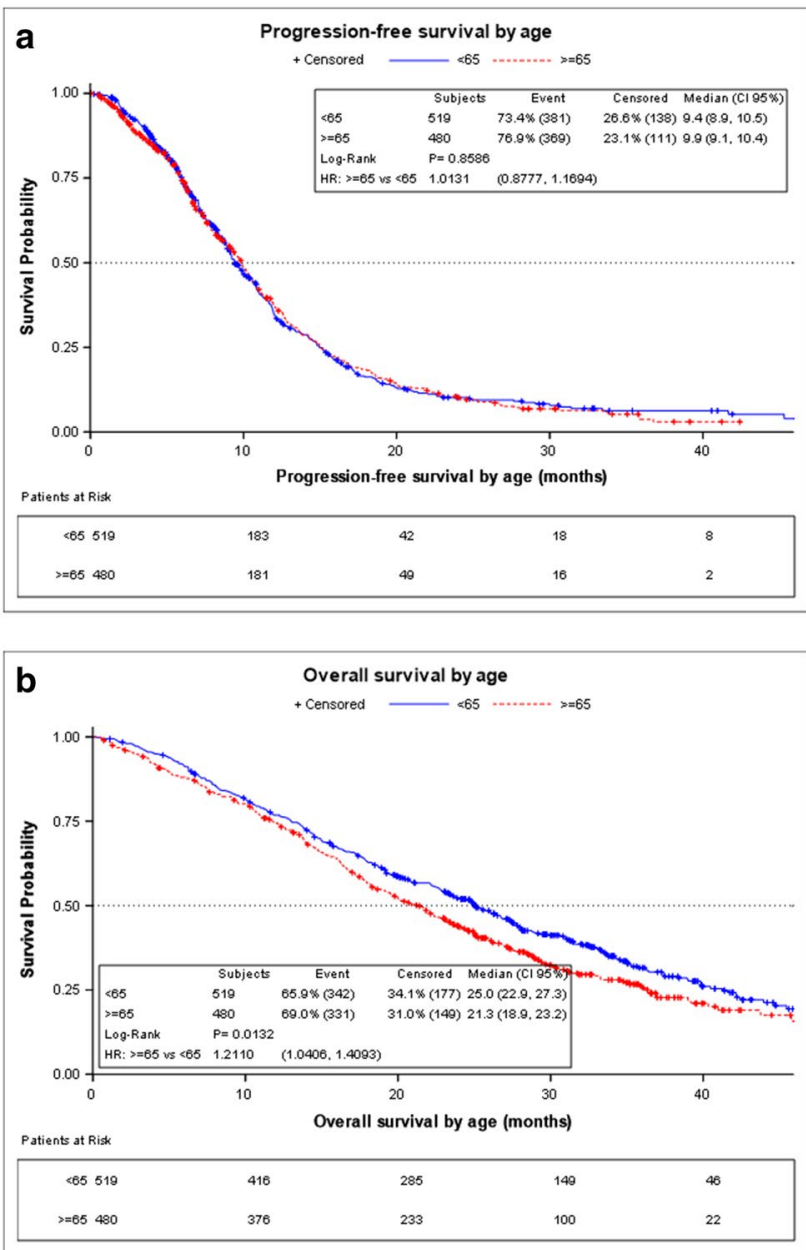

Fig. 1 Kaplan-Meier curves showing a progression-free survival and b overall survival by patient age group ( $<65$ vs. $\geq 65$ years). These plots were calculated from the derived variables in each study. $C I$ confidence interval, $H R$ hazard ratio

(72.08 vs. $63.97 \%, p=0.0061)$, as were treatment-related grade 3 or higher AEs (61.67 vs. $45.86 \%$; $p<0.0001$ ). Treatment-related serious AEs occurred in significantly more patients aged $\geq 65$ than $<65$ years $(20.83$ vs. $13.68 \% ; p=0.0027)$; corresponding values for any serious $\mathrm{AE}$ were 37.50 versus $33.33 \%(p=0.1687)$.

\subsection{Post-Study Treatment}

Significantly more patients aged $<65$ years received further chemotherapy and/or radiotherapy after discontinuing study treatment ( 76.30 vs. $68.75 \% ; p=0.0075$ ). In both age groups, the most common post-study treatment was irinotecan, followed by 5 -fluorouracil (Table 6). Other common post-study treatments (i.e. received by $>$ $20 \%$ of patients) were bevacizumab, capecitabine, cetuximab and oxaliplatin. Post-study surgery was also significantly more common in patients aged $<65$ than $\geq 65$
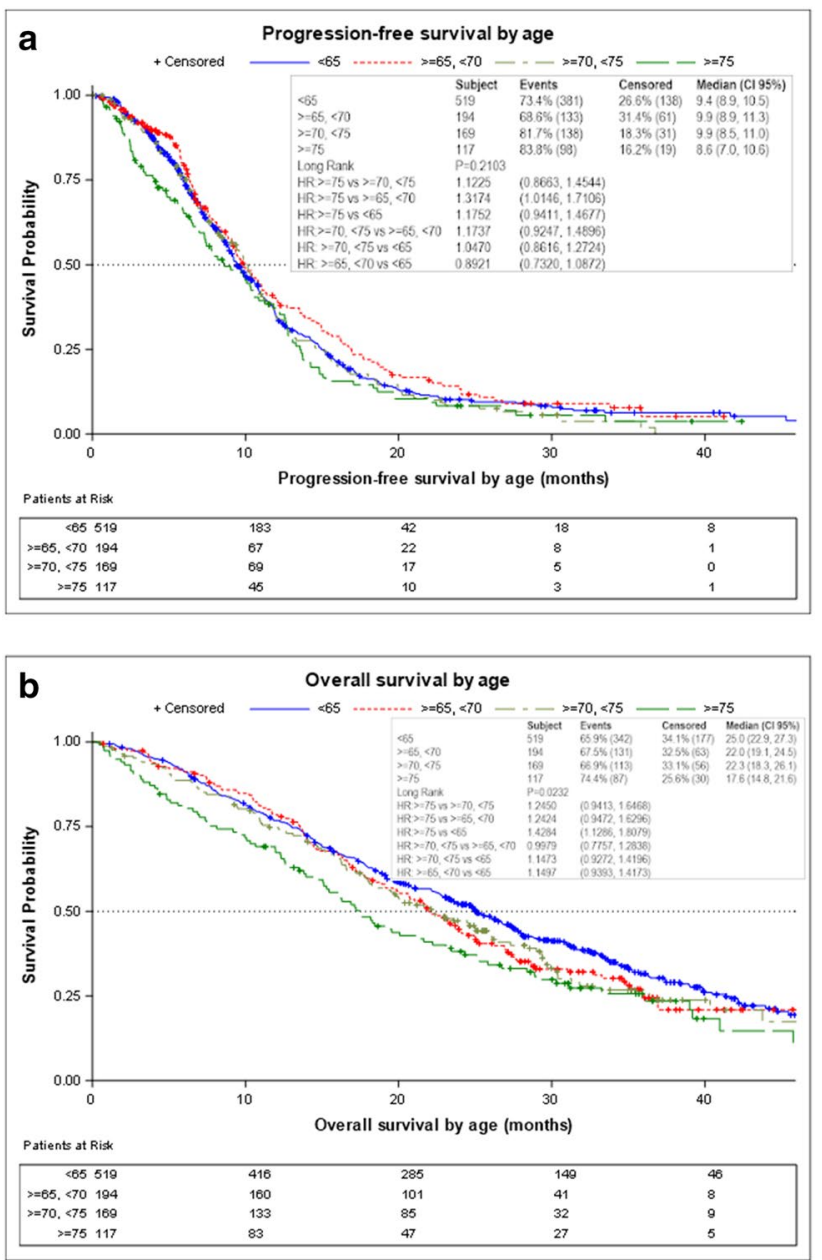

Fig. 2 Kaplan-Meier curves showing a progression-free survival and b overall survival by detailed age group. $C I$ confidence interval, $H R$ hazard ratio

years ( 24.28 vs. $16.04 \% ; p=0.0012)$. There were no significant differences between age groups in the proportions of patients who achieved R0 (no cancer cells at resection margin) or R1-R2 (microscopic or macroscopic positive margin, respectively) following surgery (Table 6).

\section{Discussion}

This large retrospective subgroup analysis of five multicentre studies of biological therapies suggests that biological therapy in combination with chemotherapy is an effective first-line treatment option for selected patients aged $\geq 65$ years with mCRC. As seen in this analysis, bevacizumabbased chemotherapy was generally the treatment used in most patients aged $\geq 65$ years ( 81.67 vs. $10.42 \%$ treated with cetuximab and $7.92 \%$ with panitumumab). PFS and ORR did 
Table 4 Efficacy endpoints by age group

\begin{tabular}{lccc}
\hline Endpoint & $\begin{array}{c}<65 \text { years } \\
(N=519)\end{array}$ & $\begin{array}{c}\geq 65 \text { years } \\
(N=480)\end{array}$ & $\begin{array}{l}\text { Total } \\
(N=999)\end{array}$ \\
\hline Median PFS, months (95\% CI) & $9.4(8.9-10.5)$ & $9.9(9.1-10.4)$ & - \\
Median OS, months (95\% CI) & $25.0(22.9-27.3)$ & $21.3(18.9-23.2)$ & - \\
Best overall response & & & $0.8586^{\mathrm{a}}$ \\
CR & $19(3.66)$ & $17(3.54)$ & $36(3.60)$ \\
PR & $303(58.38)$ & $266(55.42)$ & $569(59.96)$ \\
SD & $131(25.24)$ & $122(25.42)$ & $253(25.33)$ \\
PD & $36(6.94)$ & $33(6.88)$ & $69(6.91)$ \\
Not evaluable & $28(5.39)$ & $40(8.33)$ & $68(6.81)$ \\
Data missing & $2(0.39)$ & $2(0.42)$ & $4(0.40)$ \\
Objective response & $322(62.04)$ & $283(58.96)$ & $605(60.56)$ \\
Disease control & $453(87.28)$ & $405(84.38)$ & $858(85.89)$ \\
\hline
\end{tabular}

Data are presented as $N(\%)$ unless otherwise specified. $p<0.05$ is considered statistically significant

$C I$ confidence interval, $C R$ complete response, $O S$ overall survival, $P D$ progressive disease, $P F S$ progression-free survival, $P R$ partial response, $S D$ stable disease

${ }^{\text {a }}$ Log-ranked test

${ }^{\mathrm{b}}$ Chi-squared test

${ }^{\mathrm{c}}$ Fisher's exact test

Table 5 Most common adverse events ( $>5 \%$ of patients) for which there was a significant difference in frequency between age groups ( $<65$ and $\geq 65$ years)

\begin{tabular}{|c|c|c|c|c|c|c|c|c|c|}
\hline \multirow[t]{2}{*}{ Adverse event } & \multicolumn{3}{|l|}{ Any grade } & \multicolumn{3}{|l|}{ Grade $1-2$} & \multicolumn{3}{|l|}{ Grade $3-5$} \\
\hline & $<65$ years & $\geq 65$ years & $p$ value $^{\mathrm{a}}$ & $<65$ years & $\geq 65 \mathrm{~s}$ & $p$ value $^{\mathrm{a}}$ & $<65$ years & $\geq 65$ years & $p$ value $^{\mathrm{a}}$ \\
\hline naemia & $166(12.72)$ & $42(8.75)$ & 0.0437 & $64(12.33)$ & $42(8.75)$ & 0.0663 & $6(1.16)$ & $2(0.42)$ & 0.1902 \\
\hline Neutropenia & $110(21.19)$ & $86(17.92)$ & 0.1924 & $101(19.46)$ & $66(13.75)$ & 0.0157 & $50(9.63)$ & $48(10.00)$ & 0.8459 \\
\hline iarrhoea & $328(63.20)$ & $306(63.75)$ & 0.8565 & $318(61.27)$ & $282(58.75)$ & 0.4162 & $51(9.83)$ & $82(17.08)$ & 0.0007 \\
\hline Nausea & $220(42.39)$ & 187 (38.96) & 0.5272 & $219(42.20)$ & $5(0.96)$ & 0.2978 & $5(0.96)$ & $18(3.75)$ & 0.0033 \\
\hline Rectal haemorrhage & $31(5.97)$ & $31(6.46)$ & 0.7508 & $31(5.97)$ & $29(6.04)$ & 0.9636 & 0 & $4(0.83)$ & 0.0372 \\
\hline Vomiting & $190(36.61)$ & $168(35.00)$ & 0.5962 & $186(35.84)$ & 155 (32.29) & 0.2376 & $16(3.08)$ & $29(6.04)$ & 0.0243 \\
\hline Asthenia & $205(39.50)$ & $157(32.71)$ & 0.0257 & $202(38.92)$ & $151(31.46)$ & 0.0137 & $23(4.43)$ & $35(7.29)$ & 0.0534 \\
\hline Fatigue & $137(26.40)$ & $171(35.63)$ & 0.0016 & $136(26.20)$ & $162(33.75)$ & 0.0092 & $9(1.73)$ & $34(7.08)$ & $<0.0001$ \\
\hline URTI & $22(4.24)$ & $48(10.00)$ & 0.0004 & $22(4.24)$ & $45(9.38)$ & 0.0012 & $1(0.19)$ & $3(0.63)$ & 0.2797 \\
\hline Decreased appetite & $123(23.70)$ & $166(34.58)$ & 0.0002 & $116(22.35)$ & $160(33.33)$ & 0.0001 & $10(1.93)$ & $18(3.75)$ & 0.0811 \\
\hline Skin toxicity dermatitis ${ }^{b}$ & $152(29.29)$ & $85(17.71)$ & $<0.0001$ & $152(29.29)$ & $84(17.50)$ & $<0.0001$ & $29(5.59)$ & $14(2.92)$ & 0.0425 \\
\hline Skin fissures & $28(5.39)$ & $12(2.50)$ & 0.0197 & $27(5.20)$ & $11(2.29)$ & 0.0163 & $2(0.39)$ & $4(0.83)$ & 0.3599 \\
\hline Hypertension & $78(15.03)$ & $97(20.21)$ & 0.0314 & $72(13.87)$ & $87(18.13)$ & 0.0664 & $14(2.70)$ & $22(4.58)$ & 0.1101 \\
\hline
\end{tabular}

Data are presented as $N(\%)$ unless otherwise specified. $p<0.05$ is considered statistically significant

URTI upper respiratory tract infection

${ }^{\text {a }}$ Values in bold indicate statistically significant effect estimates at $p<0.05$

${ }^{\mathrm{b}}$ Includes dermatitis acneiform, exfoliative rash and rash

not significantly differ among patients aged $\geq 65$ versus $<$ 65 years treated with bevacizumab. A significantly higher proportion of patients aged $<65$ years received subsequent chemotherapy and/or radiotherapy, or underwent surgery, after discontinuing study treatment.
Age is known to be a major risk factor for mCRC. Both the incidence of mCRC and the number of older patients requiring treatment is expected to increase as the population of the developed world ages [12]. However, as previously mentioned, older patients are generally under-represented in clinical trials of patients with mCRC $[5,13]$. Furthermore, 
older patients may also be undertreated or remain untreated in clinical practice due to physicians' concerns about their suitability for treatment [12]. A meta-analysis of clinical trials in selected older patients with mCRC indicated that the use of biological therapy significantly improved PFS and OS in patients aged $\geq 65$ years compared with treatment that did not contain biological agents [14]. However, the available data did not allow for a between-group comparison of treatment-related toxicities in older versus younger patients [14].

Of the 999 patients included in our analysis, almost half were aged $\geq 65$ years; $17 \%$ were aged $\geq 70$ years and $12 \%$ were aged $\geq 75$ years. By pooling data from these five trials, the number of patients analysed was high enough to detect differences between patients aged $<65$ years and those aged $\geq 65$ years. However, the MACRO2 study, which excluded patients aged $>70$ years [8], contained a lower proportion of patients aged $\geq 65$ years than the other four studies. In addition, significant differences between patients aged $<65$ versus $\geq 65$ years in some baseline characteristics, including the proportion of patients with wild-type $K R A S$ tumours (74.15 vs. $63.22 \%)$ and those receiving cetuximab (26.78 vs. $10.42 \%$ ), were possibly due to inclusion criteria of the MACRO2 study, in which patients with wild-type KRAS tumours received cetuximab-based treatment [8].

The anti-tumour activity of the biological agents studied (i.e. bevacizumab, cetuximab and panitumumab) was similar in older and younger patients, with no significant differences between age groups in ORR or DCR. There were also no significant differences in median PFS between patients

Table 6 Post-treatment therapies by age group and detailed age group

\begin{tabular}{|c|c|c|c|c|c|c|}
\hline Variable & $\begin{array}{l}<65 \text { years } \\
(N=519)\end{array}$ & $\begin{array}{l}\geq 65 \text { years } \\
(N=480)\end{array}$ & $\begin{array}{l}\geq 65 \text { to }<70 \text { years } \\
(N=194)\end{array}$ & $\begin{array}{l}\geq 70 \text { to }<75 \text { years } \\
(N=169)\end{array}$ & $\begin{array}{l}\geq 75 \text { years } \\
(N=117)\end{array}$ & $\begin{array}{l}\text { Total } \\
(N=999)\end{array}$ \\
\hline \multicolumn{7}{|c|}{ Did the patient receive post-treatment therapies? } \\
\hline Yes & $396(76.3)$ & $330(68.75)$ & $137(70.6)$ & $123(72.8)$ & $70(59.8)$ & $726(72.7)$ \\
\hline No & $123(23.7)$ & $150(31.25)$ & $57(29.4)$ & $46(27.2)$ & $47(40.2)$ & $273(27.3)$ \\
\hline \multicolumn{7}{|l|}{ Therapies received } \\
\hline \multicolumn{7}{|l|}{ Chemotherapy } \\
\hline Fluoropyrimidine & $462(89.02)$ & $349(72.71)$ & $147(75.77)$ & $129(76.33)$ & $73(62.39)$ & $811(81.18)$ \\
\hline Oxaliplatin & $208(40.08)$ & $144(30.00)$ & $66(34.02)$ & $55(32.54)$ & $23(19.66)$ & $352(35.24)$ \\
\hline Irinotecan & $317(61.08)$ & $250(52.08)$ & $108(55.67)$ & $87(51.48)$ & $55(47.01)$ & $567(56.76)$ \\
\hline TAS-102 & $1(0.19)$ & $1(0.20)$ & $0(0.00)$ & $0(0.00)$ & $1(0.85)$ & $2(0.20)$ \\
\hline Mitomycin & $29(5.59)$ & $18(3.75)$ & $10(5.15)$ & $6(3.55)$ & $2(1.71)$ & $47(4.70)$ \\
\hline Gemcitabine & $10(1.93)$ & $5(1.04)$ & $1(0.52)$ & $4(2.37)$ & $0(0.00)$ & $15(1.50)$ \\
\hline Raltitrexed & $39(7.51)$ & $18(3.75)$ & $11(5.67)$ & $7(4.14)$ & $0(0.00)$ & $57(5.71)$ \\
\hline \multicolumn{7}{|l|}{ Anti-EGFR agents } \\
\hline Cetuximab & $167(32.18)$ & $117(24.38)$ & $57(29.38)$ & $35(20.71)$ & $25(21.37)$ & $284(28.43)$ \\
\hline Panitumumab & $43(8.29)$ & $39(8.13)$ & $16(8.25)$ & $15(8.88)$ & $8(6.84)$ & $82(8.21)$ \\
\hline \multicolumn{7}{|l|}{ Anti-angiogenic agents } \\
\hline Bevacizumab & $202(38.92)$ & $143(29.79)$ & $61(31.44)$ & $58(34.32)$ & $24(20.51)$ & $345(34.53)$ \\
\hline Regorafenib & $16(3.08)$ & $5(1.04)$ & $2(1.03)$ & $2(1.18)$ & $1(0.85)$ & $21(2.10)$ \\
\hline Ramucirumab & $4(0.77)$ & $6(1.25)$ & $1(0.52)$ & $2(1.18)$ & $3(2.56)$ & $10(1.00)$ \\
\hline Aflibercept & $41(7.90)$ & $18(3.75)$ & $12(6.19)$ & $4(2.37)$ & $2(1.71)$ & $59(5.91)$ \\
\hline \multicolumn{7}{|l|}{ Other } \\
\hline Others & $16(3.08)$ & $5(1.04)$ & $4(2.06)$ & $0(0.00)$ & $1(0.85)$ & $21(2.10)$ \\
\hline Received post-treatment surgery & $126(24.28)$ & $77(16.04)^{\mathrm{a}}$ & $42(21.65)$ & $22(13.02)$ & $13(11.11)$ & $203(20.32)$ \\
\hline Best surgical outcome & $N=126$ & $N=76^{\mathrm{b}}$ & $N=42$ & $N=22$ & $N=12^{\mathrm{b}}$ & $N=202^{\mathrm{b}}$ \\
\hline R0 & $84(66.67)$ & $52(68.42)$ & $30(71.43)$ & $15(68.18)$ & $7(58.33)$ & $136(13.61)$ \\
\hline $\mathrm{R} 1-\mathrm{R} 2$ & $39(30.95)$ & $19(25.00)$ & $9(21.43)$ & $6(27.27)$ & $4(33.33)$ & $58(5.81)$ \\
\hline $\mathrm{NE}$ & $3(2.38)$ & $5(6.58)$ & $3(7.14)$ & $1(4.55)$ & $1(8.33)$ & $8(0.80)$ \\
\hline
\end{tabular}

Data are presented as $N(\%)$ unless otherwise specified

$E G F R$ epidermal growth factor receptor, $N E$ not evaluable, $R O$ no cancer cells at resection margin, $R 1$ microscopic positive margin, $R 2$ macroscopic positive margin, TAS102 trifluridine/tipiracil

${ }^{\mathrm{a}} p=0.0012$ vs. $<65$

${ }^{\mathrm{b}}$ Surgical outcome data missing for one patient 
aged $\geq 65$ years $(9.9$ months $)$ and those aged $<65$ years (9.4 months). However, as may be expected, the median OS was slightly shorter in older versus younger patients (21.3 vs. 25.0 months). This OS difference may be related to the fact that significantly more patients in the younger age group underwent post-study treatment or surgery. Furthermore, different factors may have contributed to a shorter OS in older patients. First, a higher proportion of patients in that age group had worse ECOG PS. Second, a low percentage of patients received active treatment after first-line therapy. Third, the life expectancy of older patients, mainly those aged $\geq 75$ years, is usually shorter because of non-cancerspecific mortality.

Several differences were observed between older and younger patients in the safety profile of the studied drugs. Any-grade fatigue, upper respiratory tract infection, decreased appetite and hypertension occurred with significantly higher incidence among patients in the older versus younger age group. Serious or grade 3-5 treatment-related AEs and grade 3-5 fatigue and gastrointestinal disorders also occurred more frequently among older versus younger patients. In contrast, younger patients reported higher incidences of anaemia, asthenia, skin toxicities, skin fissures and mild-to-moderate neutropenia than patients in the older age group.

The extent to which differences in safety and tolerability between the age groups were attributable to biological agents or to chemotherapy remains unclear. The higher incidence of hypertension in older patients is consistent with previous studies of older patients with mCRC, in which hypertension, proteinuria and thromboembolic events were shown to be associated with bevacizumab treatment [15-17]. However, the safety of bevacizumab has previously been reported to be acceptable in patients aged $\geq 75$ years with normal scores for independent activities of daily living [18].

In general, clinical trials may not capture data on outcomes that are of particular importance in older populations, such as functional and cognitive impairment and other aspects of health-related quality of life. Additionally, less severe or less serious AEs may have more significance for, or a greater impact on, older versus younger patients, but current methodologies do not adequately address this. Thus, we acknowledge that our data may not reflect the full spectrum of adverse experiences associated with pharmacotherapy for mCRC in older patients. In future, the design of clinical trials in older adults with $\mathrm{mCRC}$ should give due consideration to patient-reported outcomes that are of specific relevance and importance to this population.

Research into the efficacy and safety of biological agents in older patients with mCRC began over 10 years ago. In 2010, a pooled analysis of four randomized studies (three first line and one second line) of bevacizumab in 3007 older patients with mCRC found that bevacizumab plus chemotherapy produced survival benefits in patients aged $\geq 65$ or $\geq 70$ years that were similar to those in patients aged $<$ 65 years [17]. Safety analyses did not identify any major additional risks associated with the use of bevacizumab in older patients, with the exception of arterial thromboembolic events, which, as expected, were more common in older than younger bevacizumab-treated patients.

Subsequently, three studies (AGITG-MAX, AVEX and PRODIGE-20) were undertaken to investigate the efficacy and safety of bevacizumab (plus a fluoropyrimidine) as firstline therapy in patients aged $\geq 75$ years with mCRC [19-21]; the results of these studies, as well as data from an earlier study in patients aged $\geq 70$ years [22], were included in a meta-analysis published in [23]. In this analysis, the addition of bevacizumab to fluoropyrimidine therapy improved both OS and PFS compared with fluoropyrimidine treatment alone, with odds ratios (ORs) of 0.78 (95\% CI 0.63-0.96) for OS and 0.55 (95\% CI 0.44-0.67) for PFS. Indeed, adding bevacizumab to fluoropyrimidine was more effective in older patients, in terms of impact on OS and PFS, than adding either irinotecan (OR 1.01 and 0.82 , respectively) or oxaliplatin (OR 0.99 and 0.81, respectively).

Because the meta-analysis considered only data from older patients, comparisons between older and younger age groups were not possible [23]. However, in the AGITGMAX trial, there was no effect of age (using a cut-off of 75 years) on PFS, OS or toxicity among patients who received bevacizumab and capecitabine [20].

Clinical trials, even those in older patients, exclude many individuals who are typical of those who present for treatment in everyday clinical practice, including those with comorbidities and renal impairment. Accordingly, data from real-world studies are needed to create a more complete picture of the effects of medical interventions in mCRC.

To date, the results of three real-world studies of biological treatments in older patients with $\mathrm{mCRC}$ have been reported [16, 24, 25]. In 2014, a prospective German observational cohort study $(n=1777)$ [25] and a retrospective population-based Czech registry study $(n=3187)$ [16] both reported that first-line bevacizumab-based regimens were effective in patients with mCRC aged $\geq 75$ and $\geq 65$ years, respectively. In the German study, median PFS and OS were shorter in older than younger patients when cut-offs of 70 and 75 years were applied [24]. In contrast, no effect of age on median PFS or OS was seen in the Czech study, which analysed data separately for patients aged $<65,65$ to $<75$ and $\geq 75$ years [16]. Lastly, in the French STROMBOLI study $(n=800)$, median PFS and OS with first-line regimens containing either bevacizumab or cetuximab were similar for patients aged $<75$ or $>5$ years and were also generally similar to those observed in our analysis. In contrast to our findings, the STROMBOLI study reported no significant 
differences between age groups in the frequency of grade 3 or 4 AEs, including gastrointestinal disorders. However, the number of patients aged $\geq 75$ years in the STROMBOLI study was relatively low $(n=126)$ compared with those aged $<75$ years $(n=674)$ [25]; thus, between-group differences may have been less apparent.

Compared with bevacizumab, less information is available on the clinical effects of cetuximab or panitumumab in the first-line treatment of older patients with mCRC. A retrospective analysis of data from the ObservEr study ( $n$ =225) demonstrated similar PFS, DCR, quality of life and incidence of AEs, with first-line chemotherapy plus cetuximab in patients with mCRC aged $<70$ versus $\geq 70$ years, suggesting that this regimen may be suitable in older patients with mCRC [26]. In addition, a retrospective analysis of observational data from the Hellenic Oncology Research Group's database suggested that panitumumab was effective (median PFS 9.4 months; median OS 23.0 months) and well-tolerated in patients with $\mathrm{mCRC}$ aged $\geq 65$ years $(n$ $=110$ ) [27]. This finding was supported by the results of the prospective phase II PANDA trial [28], in which 185 patients aged $\geq 70$ years received panitumumab in combination with folinic acid/5-fluorouracil, with or without oxaliplatin. The median PFS was 9.6 months among oxaliplatin recipients versus 9.1 months for those randomised to folinic acid/5-fluorouracil only.

Overall, the results of these studies suggest that biological-based first-line treatment of mCRC is effective in selected older patients with mCRC, and age per se should not be considered a barrier to its use.

One limitation of our study is that the data came from selected clinical trials and not from the general population/real-world studies. Therefore, the exclusion criteria of the analysed trials meant that only relatively fit older patients were included. While a higher number of older than younger patients had a baseline ECOG PS of $>0$, almost all patients in our analysis had a baseline ECOG PS of $\leq 2$. Therefore, the findings cannot be extrapolated to a population of pre-frail or frail older patients or those with poor ECOG PS. Finally, this is a retrospective study, and the MACRO2 study included in this analysis excluded patients aged $>70$ years, further introducing bias in these results.

Definitive conclusions about the relative efficacy and safety of biological agents in older versus younger patients can only be drawn from appropriately designed prospective clinical trials and meta-analyses, and our findings should be viewed in that context. We defined the boundary between 'older' and 'younger' patients as being 65 years, and this is of course an arbitrary threshold. People aged $\geq$ 65 years form a heterogeneous group, and our results cannot be extrapolated to all patients in this age category. Of note, the proportion of patients in our study who were aged $\geq 75$ years was relatively small $(<12 \%)$. We nevertheless believe our results make a useful contribution to the field of knowledge in this area and may be instructive to others conducting similar research as well as to practising geriatric oncologists.

\section{Conclusions}

This large retrospective subgroup analysis suggests that biological agents in combination with chemotherapy constitute an effective first-line treatment option for selected patients aged $\geq 65$ years with $\mathrm{mCRC}$, with efficacy comparable to that observed in patients aged $<65$ years. Toxicity is increased but generally manageable.

Supplementary Information The online version contains supplementary material available at https://doi.org/10.1007/s40266-021-00834-w.

Acknowledgements Study chairs: Dr Pilar García-Alfonso, Dr Enrique Aranda Aguilar, Dr Eduardo Díaz Rubio, Dr Javier Sastre, Dr Albert Abad and Dr Alfredo Carrato. The authors thank the medical and nursing staff at all the participating institutions, the patients and their families for participating in the study and the study investigators for their cooperation and support. A. Abad, JL Manzano (ICO. H. Germans Trias i Pujol, Badalona); A. Antón, V. Alonso Orduña (H. Miguel Servet, Zaragoza); A. Arrivi, E. Falcó (F. H. Son Llatzer, Mallorca); A. Carrato, F. Longo-Muñoz, C. Guillén-Ponce, J. L. García López (H. Ramón y Cajal, Madrid); J. Gallego, A. Rodriguez Lescure (H. General Universitario de Elche, Alicante); A. Cervantes (H. Clínico de Valencia); A. Etxeberría (Instituto Oncológico, San Sebastián); A. La Casta (H. de Donostia, San Sebastián); A. López-Ladrón (H. Nuestra Señora de Valme, Sevilla); A. M ${ }^{a}$ García Tapiador (C. H. La Mancha Centro, Ciudad Real); A. Ruiz (H. de Fuenlabrada, Madrid); A. Salud (H. de Lleida Arnau de Vilanova, Lérida); A. Sánchez Ruiz, M. Provencio (H. Puerta de Hierro, Madrid); A. Velasco (H. Universitario de la Princesa, Madrid); A. Viudez, V. Arrazubi (H. de Navarra, Pamplona); B. Massutí, A. Yuste (H. General Universitario de Alicante); B. Queralt, X. Hernández-Yagüe (ICO. H. U. Josep Trueta, Gerona); C. Alonso (H. General de Albacete); C. Fernández-Martos (Instituto Valenciano de Oncología); C. García-Girón (H. Universitario de Burgos); C. Grávalos, L. Robles (H. 12 de Octubre, Madrid); C. Martín, S. Albiol (H. del Espíritu Santo, Barcelona); C. Pericay (C. S. Parc Taulí, Barcelona); D. Almenar (H. Dr. Peset, Valencia); E. Aranda, A. Gómez (H. Universitario Reina Sofía, Córdoba); E. Barrajón (H. Clínica Benidorm, Alicante); E. Cabrera, L. del Río, A. Oltra (H. Virgen de los Lirios, Alicante); E. Díaz-Rubio, J. Sastre, B. García-Paredes (H. Universitario Clínico San Carlos, Madrid); E. González, V. Conde (H. Virgen de las Nieves, Granada); E. Jiménez (H. Jerez de la Frontera, Cádiz); E. Marcuello (H. Santa Creu i Sant Pau, Barcelona); F. Losa (H. General de L'Hospitalet, Hospitalet de Llobregat); F. Rivera, C. López-López (H. Marqués de Valdecilla, Santander); FJ. Dorta, R. Afonso (H. Ntra. Sra. de Candelaria, Tenerife); H. Manzano (H. Son Espases, Palma de Mallorca); J. Alcaide (H. Costa del Sol, Málaga); J. Alfaro (C. Sanitari de Terrasa); J. Cassinello (H. Universitario de Guadalajara); J. García-Foncillas (C. Universitaria de Navarra); J. L. García Puche (H. Clínico San Cecilio, Granada); J. M. Campos, V. Alberola (H. Arnau de Vilanova, Valencia); J. Mª Tabernero (H. Universitari Vall d'Hebrón, Barcelona); J. Ma . Vicent, A. Galán (H. de Sagunto, Valencia); J. Mª Viéitez (H. Universitario Central de Asturias, Oviedo); J. Remón (H. de Mataró, Barcelona); JL. Martín Valadés (Fundación Jiménez Díaz, 
Madrid); M. Benavides (H. Regional Universitario y Virgen de la Victoria, Málaga); M. Centelles (H. Universitari Sagrat Cor, Barcelona); M. Chaves (H. Virgen del Rocío, Sevilla); M. Constenla (Complejo Hospitalario Pontevedra); M. Gallén, C. Montagut (H. del Mar, Barcelona); M. Llanos (H. Universitario de Canarias, Tenerife); M. Méndez Ureña (H. de Móstoles, Madrid); M. Navalón (Complejo Asistencial de Zamora); M. Nogué, M. Gay (H. General de Vic, Barcelona); M. Valladares, M. Reboredo (C. H. Universitario, La Coruña); M $^{\mathrm{a}}$ J. Gómez (H. Puerta del Mar, Cádiz); Ma J. Safont (H. General Universitario, Valencia); P. Bueso (H. de Barbastro, Huesca); P. Escudero (H. C. Universitario Lozano Blesa, Zaragoza); P. García Alfonso (H. Universitario Gregorio Marañón, Madrid); P. García-Teijido (H. San Agustín, Avilés); P. Martínez de Prado (H. de Basurto, Bilbao); R. Dueñas, N. Cárdenas (H. Ciudad de Jaén); R. Grández (H. Reina Sofía de Tudela); R. López López (H. Clínico Universitario de Santiago); R. Salazar (ICO. H. Duran i Reinals, Barcelona); R. Vera, A. Viudez (H. de Navarra, Pamplona); S. Merino (H. San Joan de Reus, Tarragona); T. Checa (I. de Oncología Corachán, Barcelona); T. García García (H. Morales Meseguer, Murcia); V. Valentí, S. Casado (H. General de Catalunya, Barcelona). We would like to thank Toni Dando and Sarah Greig, PhD, of Springer Healthcare Communications, who wrote the outline and first draft of this manuscript, respectively; and Matthew Weitz, Tracy Harrison and Alma Orts-Sebastian, $\mathrm{PhD}$, also of Springer Healthcare, for editing assistance prior to submission. We would also like to thank Inmaculada Ruiz de Mena and Susana Rodriguez at TTD Data Centre and Lucía Rodrigo (Statistics and Data Management) at Pivotal for their help.

\section{Declarations}

Funding This study was funded by the Spanish Cooperative Group for the Treatment of Digestive Tumours (TTD) collaborative group. All medical writing assistance was funded by the Spanish Cooperative TTD and Roche Farma SA.

Conflict of Interest E Díaz Rubio has received grants from SYSMEX, Merck Serono, Roche, AstraZeneca and Amgen. A Carrato is an advisory board member for Merck, Roche, MSD, Pfizer, Bayer, Shire, Servier, Celgene and Amgen and has received research funds from Celgene, Shire and Amgen. B Massutí has received payment for consulting or advisory roles from Roche, Boehringer Ingelheim, BristolMyers Squibb, Merck Sharpe and Dohme and AstraZeneca; participating in speakers' bureau from Roche, Amgen, Merck Serono, Pfizer, AstraZeneca and Boehringer Ingelheim; and travel, accommodation or expenses from Boehringer Ingelheim, Merck Sharp and Dohme, Janssen and Roche. A Muñoz has received grants from Sanofi, Celgene, Leo Pharma; non-financial support from Merck Serono; personal fees and non-financial support from Sanofi, Celgene, Roche and Amgen; and personal fees from AstraZeneca, Leo Pharma, Servier, Pfizer, Daiichi Sankyo, Bayer and Halozyme, Merck Sharp and Dohme, Rovi and Lilly. J Sastre has received honoraria for speaking roles with Merck, Roche, Ipsen, Lilly, Shire, Pfizer, Servier and MSD; advisory roles with Roche, Amgen, Merck, Bayer, Celgene, Servier, BMS, Sanofi and Ipsen; and travelling and accommodation support from Merck and Ipsen. R Ferreiro has received honoraria for speaking and advisory roles and personal fees and travelling and accommodation support from Roche, Amgen and Servier. M Valladares-Ayerbes has received grants from Roche and personal fees from Roche, Merck, Amgen, Sanofi, Servier, Bayer and Celgene. JM Viéitez has received payments for consulting or advisory roles from Roche, Amgen and Servier; travel, accommodation and expenses from Roche, Amgen, Servier and Bristol-Myers Squibb; expert testimony from Servier; and research funding from Amgen and Roche. E Aranda has received honoraria for advisory roles from Amgen, Bayer, Celgene, Merck, Roche and Sanofi.
P García Alfonso, A Abad, MJ Ortiz-Morales, JL Manzano Mozo, G Durán, MJ Safont, F Rivera, E González, C Grávalos, V AlonsoOrduña and A Yubero have no conflicts of interest that are directly relevant to the content of this article.

Ethics approval All studies included in this retrospective analysis were conducted in accordance with the Declaration of Helsinki and were approved by institutional review boards or independent ethics committees at each centre.

Consent to participate All patients gave written informed consent to participate in these studies.

Data Availability The datasets generated and analysed during the current study are available from the corresponding author on reasonable request.

Code Availability Not applicable.

Author Contributions Conception/design: P García Alfonso. Provision of study material or patients: all authors. Collection and/or assembly of data: all authors. Data analysis and interpretation: P García Alfonso. Manuscript preparation: P García Alfonso. Final approval of manuscript: all authors.

Open Access This article is licensed under a Creative Commons Attribution-NonCommercial 4.0 International License, which permits any non-commercial use, sharing, adaptation, distribution and reproduction in any medium or format, as long as you give appropriate credit to the original author(s) and the source, provide a link to the Creative Commons licence, and indicate if changes were made. The images or other third party material in this article are included in the article's Creative Commons licence, unless indicated otherwise in a credit line to the material. If material is not included in the article's Creative Commons licence and your intended use is not permitted by statutory regulation or exceeds the permitted use, you will need to obtain permission directly from the copyright holder. To view a copy of this licence, visit http://creativecommons.org/licenses/by-nc/4.0/.

\section{References}

1. Wilkins T, McMechan D, Talukder A. Colorectal cancer screening and prevention. Am Fam Physician. 2018;97(10):658-65.

2. National Cancer Institute. SEER cancer statistics review, 19752016, based on November 2018 SEER data submission, posted to the SEER web site, April 2019. Bethesda, MD. https://seer.cance r.gov/csr/1975_2016/. Accessed 12 June 2019.

3. Van Cutsem E, Cervantes A, Adam R, et al. ESMO consensus guidelines for the management of patients with metastatic colorectal cancer. Ann Oncol. 2016;27(8):1386-422.

4. Booth CM, Karim S, Mackillop WJ. Real-world data: towards achieving the achievable in cancer care. Nat Rev Clin Oncol. 2019;16(5):312-25.

5. Lewis JH, Kilgore ML, Goldman DP, et al. Participation of patients 65 years of age or older in cancer clinical trials. J Clin Oncol. 2003;21(7):1383-9.

6. Aparicio T, Canoui-Poitrine F, Caillet P, et al. Treatment guidelines of metastatic colorectal cancer in older patients from the French Society of Geriatric Oncology (SoFOG). Dig Liver Dis. 2020;52(5):493-505.

7. Garcia-Alfonso P, Chaves M, Munoz A, et al. Capecitabine and irinotecan with bevacizumab 2-weekly for metastatic colorectal cancer: the phase II AVAXIRI study. BMC Cancer. 2015;15:327. 
8. Aranda E, Garcia-Alfonso P, Benavides M, et al. First-line mFOLFOX plus cetuximab followed by mFOLFOX plus cetuximab or single-agent cetuximab as maintenance therapy in patients with metastatic colorectal cancer: phase II randomised MACRO2 TTD study. Eur J Cancer. 2018;101:263-72.

9. Carrato A, Abad A, Massuti B, et al. First-line panitumumab plus FOLFOX 4 or FOLFIRI in colorectal cancer with multiple or unresectable liver metastases: a randomised, phase II trial (PLANETTTD). Eur J Cancer. 2017;81:191-202.

10. Diaz-Rubio E, Gomez-Espana A, Massuti B, et al. First-line XELOX plus bevacizumab followed by XELOX plus bevacizumab or single-agent bevacizumab as maintenance therapy in patients with metastatic colorectal cancer: the phase III MACRO TTD study. Oncologist. 2012;17(1):15-25.

11. Abad A, Martinez-Balibrea E, Vieitez JM, et al. Genotype-based selection of treatment of patients with advanced colorectal cancer (SETICC): a pharmacogenetic-based randomized phase II trial. Ann Oncol. 2018;29(2):439-44.

12. Tapia Rico G, Townsend AR, Broadbridge V, Price TJ. Targeted therapies in elderly patients with metastatic colorectal cancer: a review of the evidence. Drugs Aging. 2017;34(3):173-89.

13. Canoui-Poitrine F, Lievre A, Dayde F, et al. Inclusion of older patients with cancer in clinical trials: the SAGE prospective multicenter cohort survey. Oncologist. 2019;24(12):e1351-9.

14. Zhao C, Li S, Liu Q. Meta-analysis of molecular targetted agents in the treatment of elderly patients with metastatic colorectal cancer: does the age matter? J Chemother. 2016;28(4):321-7.

15. Feliu J, Salud A, Safont MJ, et al. Correlation of hypertension and proteinuria with outcome in elderly bevacizumabtreated patients with metastatic colorectal cancer. PLoS ONE. 2015;10(1):e0116527.

16. Slavicek L, Pavlik T, Tomasek J, et al. Efficacy and safety of bevacizumab in elderly patients with metastatic colorectal cancer: results from the Czech population-based registry. BMC Gastroenterol. 2014;14:53

17. Cassidy J, Saltz LB, Giantonio BJ, Kabbinavar FF, Hurwitz HI, Rohr UP. Effect of bevacizumab in older patients with metastatic colorectal cancer: pooled analysis of four randomized studies. J Cancer Res Clin Oncol. 2010;136(5):737-43.

18. Aparicio T, Bouche O, Francois E, et al. Geriatric analysis from PRODIGE 20 randomized phase II trial evaluating bevacizumab + chemotherapy versus chemotherapy alone in older patients with untreated metastatic colorectal cancer. Eur J Cancer. 2018;97:16-24.

19. Cunningham D, Lang I, Marcuello E, et al. Bevacizumab plus capecitabine versus capecitabine alone in elderly patients with previously untreated metastatic colorectal cancer (AVEX): an open-label, randomised phase 3 trial. Lancet Oncol. 2013;14(11):1077-85.

20. Price TJ, Zannino D, Wilson K, et al. Bevacizumab is equally effective and no more toxic in elderly patients with advanced colorectal cancer: a subgroup analysis from the AGITG MAX trial: an international randomised controlled trial of capecitabine, bevacizumab and mitomycin C. Ann Oncol. 2012;23(6):1531-6.

21. Aparicio T, Bouché O, Taieb J, et al. Bevacizumab + chemotherapy versus chemotherapy alone in elderly patients with untreated metastatic colorectal cancer: a randomized phase II trial-PRODIGE 20 study results. Ann Oncol. 2018;29(1):133-8.

22. Kabbinavar FF, Schulz J, McCleod M, et al. Addition of bevacizumab to bolus fluorouracil and leucovorin in first-line metastatic colorectal cancer: results of a randomized phase II trial. J Clin Oncol. 2005;23(16):3697-705.

23. Landre T, Maillard E, Taleb C, et al. Impact of the addition of bevacizumab, oxaliplatin, or irinotecan to fluoropyrimidin in the first-line treatment of metastatic colorectal cancer in elderly patients. Int J Colorectal Dis. 2018;33(8):1125-30.

24. Hofheinz R, Petersen V, Kindler M, et al. Bevacizumab in firstline treatment of elderly patients with metastatic colorectal cancer: German community-based observational cohort study results. BMC Cancer. 2014;14:761.

25. Gouverneur A, Coutureau J, Jove J, et al. Patterns of use, safety, and effectiveness of targeted therapies in first-line treatment of metastatic colorectal cancer according to age: the STROMBOLI cohort study. Clin Colorectal Cancer. 2019;18(1):e150-62.

26. Rosati G, Pinto C, Di Fabio F, et al. Quality of life, compliance, safety and effectiveness in fit older metastatic colorectal patients with cancer treated in first-line with chemotherapy plus cetuximab: a restrospective analysis from the ObservEr study. J Geriatr Oncol. 2018;9(3):243-8.

27. Asimakopoulou N, Souglakos J, Kentepozidis N, et al. Efficacy of panitumumab in older patients with metastatic colorectal cancer: a retrospective analysis using the database of the Hellenic Oncology Research Group (HORG). J Geriatr Oncol. 2019;10(1):143-8.

28. Lonardi S, Schirripa M, Buggin F, et al. First-line FOLFOX plus panitumumab versus $5 \mathrm{FU}$ plus panitumumab in RAS-BRAF wildtype metastatic colorectal cancer elderly patients: the PANDA study. J Clin Oncol. 2020;38(15_suppl):4002. 


\section{Authors and Affiliations}

Pilar García-Alfonso ${ }^{1}$ (D) Eduardo Díaz-Rubio ${ }^{2}$ - Albert Abad ${ }^{3,4}$ - Alfredo Carrato ${ }^{5}$ - Bartomeu Massutí ${ }^{6}$ María José Ortiz-Morales ${ }^{7}$. José Luis Manzano Mozo ${ }^{3}$. Andrés Muñoz ${ }^{1}$. Gema Durán ${ }^{8}$. Javier Sastre ${ }^{2}$. María José Safont ${ }^{9} \cdot$ Reyes Ferreiro ${ }^{5}$. Fernando Rivera $^{10}$. Encarnación González ${ }^{11}$. Manuel Valladares-Ayerbes ${ }^{12}$. Cristina Grávalos ${ }^{13}$. Vicente Alonso-Orduña ${ }^{14}$. José María Viéitez ${ }^{15}$. Alfonso Yubero ${ }^{16}$. Enrique Aranda ${ }^{7}$ on behalf of the Spanish Cooperative Group for the Treatment of Digestive Tumors (TTD)

1 Servicio de Oncología, Instituto de Investigación Sanitaria Gregorio Marañón, Hospital General Universitario Gregorio Marañón, Madrid, Spain

2 CIBERONC, Hospital Clínico San Carlos, Instituto de Investigación Hospital Clinico San Carlos (IdISSC), Madrid, Spain

3 ICO, Hospital Germans Trias i Pujol, Badalona, Spain

4 IOR Hospital Universitario Dexeus, Barcelona, Spain

5 IRYCIS, CIBERONC, Hospital Universitario Ramón y Cajal, Alcalá University, Madrid, Spain

6 Hospital General Universitario de Alicante, Alicante, Spain

7 IMIBIC, CIBERONC, Reina Sofía Hospital, Instituto de Salud Carlos III, University of Córdoba, Córdoba, Spain

8 Hospital Universitario Regional y Virgen de la Victoria, Malaga, Spain

9 Hospital General Universitario de Valencia, Valencia, Spain
10 Hospital Universitario Marqués de Valdecilla, IDIVAL, Santander, Spain

11 Hospital Virgen de las Nieves, Granada, Spain

12 Instituto de Investigación Biomédica (INIBIC), Complejo Hospitalario Universitario A Coruña, A Coruña, Spain

13 Hospital Universitario, 12 de Octubre, Madrid, Spain

14 Instituto de Investigación Sanitaria de Aragón (IISA), Hospital Universitario Miguel Servet, Zaragoza, Spain

15 Hospital Universitario Central de Asturias, Oviedo, Spain

16 Hospital Clínico Universitario Lozano Blesa, Zaragoza, Spain 